\title{
Evaluation of preventive and therapeutic activity of novel non-steroidal anti-inflammatory drug, CG100649, in colon cancer: Increased expression of TNF-related apoptosis-inducing ligand receptors enhance the apoptotic response to combination treatment with TRAIL
}

\author{
JONG KYU WOO $^{1 *}$, JU-HEE KANG ${ }^{2 *}$, YEONG-SU JANG ${ }^{1}$, SEONGGU RO $^{3}$, JOONG MYUNG CHO ${ }^{3}$, \\ HWAN-MOOK KIM ${ }^{1}$, SANG-JIN LEE ${ }^{2}$ and SEUNG HYUN OH ${ }^{1}$ \\ ${ }^{1}$ College of Pharmacy, Gachon Institute of Pharmaceutical Science, Gachon University, Incheon 406-840; \\ ${ }^{2}$ Research Institute, National Cancer Center, Goyang-si, Gyeonggi-do 410-769; ${ }^{3}$ Crystal Genomics, \\ Seongnam-si, Gyeonggi-do 463-400, Republic of Korea
}

Received November 27, 2014; Accepted January 23, 2015

DOI: $10.3892 / o r .2015 .3793$

\begin{abstract}
Non-steroidal anti-inflammatory drugs (NSAIDs) have been suggested as the potential new class of preventive or therapeutic antitumor agents. The aim of the present study was to evaluate the antitumor activity of the novel NSAID, CG100649. CG100649 is a novel NSAID dual inhibitor for COX-2 and carbonic anhydrase (CA)-I/-II. In the present study, we investigated the alternative mechanism by which CG100649 mediated suppression of the colon cancer growth and development. The anchorage-dependent and -independent clonogenic assay showed that CG100649 inhibited the clonogenicity of human colon cancer cells. The flow cytometric analysis showed that CG100649 induced the G2/M cell cycle arrest in colon cancer cells. Animal studies showed that CG100649 inhibited the tumor growth in colon cancer xenograft in nude mice. Furthermore, quantitative PCR and FACS analysis demonstrated that CG100649 upregulated the expression of TNF-related apoptosis-inducing ligand (TRAIL) receptors (DR4 and DR5) but decreased the expression of decoy receptors (DcR1 and DcR2) in colon cancer cells. The results showed that CG100649 treatment sensitized TRAIL-mediated growth suppression and apoptotic cell death. The combination treatment resulted in significant repression
\end{abstract}

Correspondence to: Professor Seung Hyun Oh, College of Pharmacy, Gachon University, 7-45 Songdo-dong, Yeonsu-gu, Incheon 406-840, Republic of Korea

E-mail: eyeball@hanmail.net; eyeball@gachon.ac.kr

*Contributed equally

Key words: non-steroidal anti-inflammatory drugs, colon cancer, TNF-related apoptosis-inducing ligand, apoptosis of the intestinal polyp formation in $\mathrm{APC}^{\mathrm{min} /+}$ mice. Our data clearly demonstrated that CG100649 contains preventive and therapeutic activity for colon cancer. The present study may be useful for identification of the potential benefit of the NSAID CG100649, for the achievement of a better treatment response in colon cancer.

\section{Introduction}

Colon cancer is one of the most common solid tumors and frequent cause of cancer-related mortality worldwide $(1,2)$. Despite significant progress in conventional therapeutic approaches to colon cancer, most patients eventually succumb to their disease. Thus there is a requirement to develop new preventive and/or therapeutic approaches to this malignancy. Various epidemiological studies have reported that the continued use of non-steroidal anti-inflammatory drugs (NSAIDs) is associated with a significant decrease in cancer incidence and suppressed progression of this malignant disease $(3,4)$. The use of NSAIDs has also been connected with a reduced risk of cancer-related mortality and distant metastasis $(3,4)$. However, the long-term use of NSAIDs for chemoprevention generates severe side effects associated with their cyclooxygenase (COX)-inhibitory activity and suppression of prostaglandin synthesis. This requires the development of novel drugs to prevent and treat colon cancer. CG100649 is a novel NSAID drug applicant with tissue-specific activity that is designed to deliver sustained levels of the drug to cancer tissues while maintaining low systemic exposure by binding to carbonic anhydrase (CA) in red blood cells (5). Its specific dual COX-2 and CA binding properties provide superior safety to various tissues than already approved COX-1 or COX-2 inhibitor drugs.

The TNF-related apoptosis-inducing ligand (TRAIL) binds to TRAIL-R1/DR4 and TRAIL-R2/DR5 (6,7). Previous studies have shown that TRAIL is a useful molecule with a 
lot of functions in cancer and immunity. TRAIL is known to induce apoptosis in numerous cancer cell lines while exhibiting minimal or no toxicity in normal cells $(6,7)$. A variety of experimental animal studies have revealed that TRAIL has great promise as a selective anticancer agent $(7,8)$. TRAIL death receptors DR4 and DR5 aid the selective removal of malignant cells through the induction of apoptosis $(9,10)$. TRAIL induces cell death via caspase-dependent apoptosis due to binding to DR4 or DR5. Nonfunctional 'decoy' receptors, DcR1 and DcR2, compete for TRAIL binding, thereby promoting cell survival (10).

In the present study, we investigated the therapeutic and chemopreventive effect of CG100649 in colon cancer and the mechanism by which CG100649 suppresses the development of colon cancer. The results showed that CG100649 significantly increased TRAIL receptors, DR4 and DR5. CG100649 seems to exhibit its anticancer activity upregulation of TRAIL receptors, independent of inhibition activity of COX-2 and CA-1/II. Our studies show strong clinical potential since CG100649 alone or in combination with TRAIL can be used to prevent and/or treat colon cancer. Moreover, we investigated the alternative mechanism underlying the potential antitumor effect of CG100649.

\section{Materials and methods}

Cell lines and reagents. Human colon cancer cell lines (DLD-1, HCT116 and HT29) and human lung cancer cell lines (A549 and 226Br) were obtained from the American Type Culture Collection (ATCC; Manassas, VA, USA). The cell lines were cultured in RPMI-1640 supplemented with $10 \%$ fetal bovine serum (FBS) (both from Welgene, Seoul, Korea) and $1 \%$ penicillin-streptomycin (Gibco-BRL Life Technologies, Gaithersburg, MD, USA). The cell lines were cultured at $37^{\circ} \mathrm{C}$ in the presence of $5 \% \mathrm{CO}_{2}$. CG100649 was obtained from CrystalGenomics (Goyang, Korea).

Clonogenic survival assays. For the anchorage-dependent colony formation assay, cancer cells were seeded in 6-well culture plates at a density of $5 \times 10^{2}$ cells/well. After overnight incubation, the cells were treated with different concentrations $(0-100 \mu \mathrm{M})$ of CG100649 or vehicle and maintained for 10 days at $37^{\circ} \mathrm{C}$. The cells were fed after 4 days by removing old medium and adding fresh medium. The plates were then stained with hematoxylin and the colony number was counted. For the anchorage-independent colony formation assay, soft agar was used as previously described (14). Briefly, $1 \times 10^{3}$ cells were suspended in $1 \mathrm{ml}$ of $0.3 \%$ soft agar that was layered on top of $1 \mathrm{ml}$ of $0.9 \%$ solidified agar in each well of 12-well plates. The plates were then incubated for 15-21 days in complete RPMI medium containing 50 or $100 \mu \mathrm{M}$ CG100649. The medium was changed every 4 days during this period. At the end of the experiment, tumor cell colonies measuring $\geq 30 \mu \mathrm{m}$ were counted using a dissection microscope.

Cell cycle and apoptotic cell death analysis. Cells were fixed and stained according to a modified Krishan technique (11). Briefly, $2 \times 10^{5}$ cells were seeded in $60-\mathrm{mm}$ dishes. After $24 \mathrm{~h}$, the cells were treated with different concentrations of CG100649 or vehicle for $48 \mathrm{~h}$. The cells were then trypsin- ized, rinsed once, resuspended in phosphate-buffered saline (PBS), fixed with ice-cold $70 \%$ ethanol for $\geq 20 \mathrm{~min}$ and stored at $-20^{\circ} \mathrm{C}$. Fixed cells were rinsed again and re-suspended in PBS containing $50 \mu \mathrm{g} / \mathrm{ml}$ propidium iodide (PI) (Sigma, St. Louis, MO, USA) and $40 \mathrm{kU} / \mathrm{ml}$ DNase-free RNase (Stratagene, La Jolla, CA, USA). The cells were then run on a Becton-Dickinson FACScan flow cytometer (BD Biosciences, San Jose, CA, USA). Induction of apoptosis triggered by treatment in cancer cells was measured by fluorescence-conjugated Annexin V and PI co-staining (Santa Cruz Biotechnology, Inc., Santa Cruz, CA, USA). The cells were treated with CG-100649 and TRAIL for $48 \mathrm{~h}$ than resuspended in $200 \mu \mathrm{l}$ of Annexin V binding buffer containing $5 \mu \mathrm{l}$ Annexin V FITC plus $10 \mu \mathrm{l}$ of PI solution $(50 \mu \mathrm{g} / \mathrm{ml})$. The cells were submitted to cytofluorimetric analysis on a FACScan flow cytometer to differentiate the live cells (unstained) from the apoptotic ones (stained only with Annexin V and stained with both Annexin V and PI).

FACS analysis for DR4 and DR5 expression. The surface expression of the DR4 and DR5 was determined by flow cytometry. Colon cancer cells, treated with CG100649 for $48 \mathrm{~h}$, were collected and incubated with anti-DR4 (Abcam, Cambridge, MA, USA) and DR5 (Cell Signaling Technology, Danvers, MA, USA) primary antibody for $1 \mathrm{~h}$ at $4^{\circ} \mathrm{C}$ and subsequently incubated with fluorescence-conjugated secondary antibodies for $1 \mathrm{~h}$ at $4^{\circ} \mathrm{C}$. Surface staining was determined with a FACScan flow cytometer.

Quantitative PCR. Colon cancer cells were treated with or without CG100649 (20 or $50 \mu \mathrm{M}$ ) for $48 \mathrm{~h}$. Total RNA from the cells was isolated using TRIzol (Invitrogen, Carlsbad, CA, USA). For quantitative PCR (qPCR), $1 \mu \mathrm{g}$ of RNA was used as a template for reverse-transcription using the PrimeScript First Strand cDNA Synthesis kit (Takara, Kyoto, Japan). PCR was carried out with $50 \mathrm{ng}$ of cDNA using a PCR pre-mixture (Takara). Samples were run in triplicate and the relative expression of target genes was determined by normalizing each value to actin. The primer sequences used were: actin (forward, 5'-CCT CTA TGC CAA CAC AGT GC-3' and reverse, 5'-GTA CTC CTG CTT GCT GAT CC-3'); DR4 (forward, 5'-TTC TCC CGT TTG CTT ATC ACA CGC-3' and reverse, 5'-GGA CAC GGC AGA GCC TGT GCC ATC-3'); DR5 (forward, 5'-GGG AGC CGC TCA TGA GGA AGT TGG-3' and reverse, 5'-GGC AAG TCT CTC TCC CAG CGT CTC-3'); DcRl (forward, 5'-GTT TGT TTG AAA GAC TTC ACT GTG-3' and reverse, 5'-GCA GGC GTT TCT GTC TGT GGG AAC-3'); DcR2 (forward, 5'-CTT CAG GAA ACC AGA GCT TCC CTC-3' and reverse, 5'-TTC TCC CGT TTG CTT ATC ACA CGC-3').

Xenograft tumor model. Six-week-old female BALB/c nude mice were purchased from the Jackson Laboratory (Bar Harbor, ME, USA) and maintained under specific pathogen-free conditions. Briefly, HT29 colon cancer cells were subcutaneously injected $\left(1 \times 10^{7}\right.$ cells in $100 \mu \mathrm{l}$ of PBS at a single dorsal site in BALB/c nude mice. After the tumor graft, the mice were given CG100649 (5 mg/ $\mathrm{kg}$ ) five times a week for 26 days. The resulting tumors were measured once or twice/week for 7 weeks. Volumes were calculated by the formula: $0.5 \mathrm{x} \mathrm{a} \mathrm{x} \mathrm{b}^{2}$, where $\mathrm{a}$ and $\mathrm{b}$ are the longer and shorter 
diameters, respectively. Animal care and experimental procedures were approved by the Institutional Animal Care and Use Committee at the National Cancer Center, Korea.

$A P C^{\text {min/t }}$ mice spontaneous colon cancer model. C57BL/ $6 \mathrm{~J}-\mathrm{APC}^{\mathrm{min} /+}$ mice were purchased from Jackson Laboratory. Heterozygote of these strains and wild-type (C57BL/6J) were prepared for the experiments. One week after acclimatization, $\mathrm{APC}^{\mathrm{min} / \mathrm{+}}$ mice were divided into four groups of 10 animals (5 mice/gender) each and given $0.2 \mathrm{ml}$ vehicle $(0.5 \% \mathrm{w} / \mathrm{v}$ carboxymethyl cellulose and $0.025 \%$ Tween-20 in distilled water) or $1 \mathrm{mg} / \mathrm{kg}$ CG100649 by per os (p.o.) gavage in $0.2 \mathrm{ml}$ vehicle once a week for 14 weeks. From the third week, the mice were given TRAIL ( $3 \mathrm{mg} / \mathrm{kg}$ ) twice a week for 12 weeks. Animal care and treatments were in accordance with the approved protocol and institutional guidelines. The experiment was terminated after the 14-week treatment period (at 20 weeks of age) to minimize the mortality risk caused by severe anemia and intestinal obstruction, which is more common in $\mathrm{APC}^{\text {min/t }}$ mice at this age. Following euthanasia, the small intestine and colon were removed, opened longitudinally and rinsed with saline. The small intestine was divided by length into the proximal, middle and distal segments and spread onto microscope slides. Polyps on intestinal segments including colon were counted and their sizes were measured with a digital caliper under a dissecting microscope.

Immunohistochemistry and TUNEL staining. Immunohistochemical assays and TUNEL staining were carried out as previously described $(12,13)$. Tissues in paraffin block were sectioned at $4-\mu \mathrm{m}$ thickness. Deparaffinization antigen retrieval was performed using citrate buffer $[10 \mathrm{mM}$ citrate and $0.05 \%$ Tween- 20 (pH 6.0)] and the sections were heated in a pressure cooker for $25 \mathrm{~min}$ and then cooled to room temperature. Blocking of endogenous peroxides was accomplished by incubating sections in $3 \%$ hydrogen peroxide in PBS for $30 \mathrm{~min}$. The sections were incubated with primary antibodies against DR4 or DR5 (Thermo Scientific, Waltham, MA, USA) overnight at $4^{\circ} \mathrm{C}$ and then processed with a biotinylated secondary antibody followed by the addition of avidin/ biotin complex (Vector Laboratories, Burlingame, CA, USA). Diaminobenzidine (Dako A/S, Glostrup, Denmark) was used as a chromogen and commercial hematoxylin was used for counterstaining. Another part was sectioned for terminal deoxynucleotidyl transferase-mediated UTP end-labeling (TUNEL) staining, a measure of apoptosis. The TUNEL assay was performed as previously described $(13,14)$. For apoptotic analysis, nuclei in the field and TUNEL-positive cells were counted in 3-5 randomly selected fields of view at a magnification of $\mathrm{x} 200$.

Statistical analysis. Experiments were carried out in duplicate or triplicate. A two-tailed Student's t-test was used for statistical analysis of comparative data using Microsoft Excel software (Microsoft Co., Tokyo, Japan). P $<0.05$ was considered significant and indicated by asterisks in the images. For the graphical representation of data indicated, the y-axis error bars were presented as standard deviation (SD) of the data for each point marked on the graph.

\section{Results}

CG100649 inhibits survival and cell cycle progression of human colon and lung cancer cells. The growth suppressive activity of CG100649 was investigated in human colon and lung cancer cells using clonogenic survival assays (anchoragedependent and -independent). As shown in Fig. 1A, CG100649 dose-dependently inhibited the anchorage-dependent colony formation in colon cancer cells (HT-29, DLD-1 and HCT116) and lung cancer cells (A549). It was found that $10 \mu \mathrm{M}$ concentrations of CG100649 caused significant inhibition of colony formation in HT-29 (22.6\%, p<0.05, Fig. 1A). Consistent with this result, CG100649 strongly decreased anchorage-independent colony formation in DLD-1, HCT116, A549 and 226Br cells (Fig. 1B). These results suggested that CG100649 has growth-inhibitory activity in colon and lung cancer cells. We evaluated the capacity of CG100649 on cell cycle progression to determine whether the CG100649-mediated inhibition of proliferation was associated with cell cycle regulation. Histograms of DNA content were obtained from PI-stained cancer cells exposed to CG100649. As shown in Fig. 2, CG100649 accumulated the cell population in S and G2/M phase but decreased it in G1 phase suggesting that CG100649 arrests the cell cycle in the G2/M phase in all the cancer cells. The cells accumulated at the $\mathrm{S}$ and G2/M phase at a relatively lower concentration of CG100649 and at the G2/M phase at a relatively higher concentration of CG100649 when they were treated with an increasing amount of CG100649.

CG100649 inhibits xenograft tumor growth in Balb/c nude mice. HT-29 colon cancer cells were injected into Balb/c nude mice at the right side of the posterior lateral trunk for tumor nodule generation to determine the effect of CG100649 on tumor growth in vivo. From the 6th day, we treated mice with CG100649 or vehicle for 20 days. Each tumor was measured in two dimensions with electric calipers twice weekly for 3 weeks and the tumor volume was calculated. Treatments were highly tolerated, and no significant weight loss was observed (Fig. 3C). We found that CG100649 decreased tumor volume as compared to vehicle control; the average tumor volume of CG100649-treated tumors at study termination was $245.9 \pm 50.2 \mathrm{~mm}^{3}$ as compared to $382.0 \pm 71.9 \mathrm{~mm}^{3}$ for controls ( $<<0.001$; Fig. 3B) suggesting a therapeutic effect of CG100649 on colon cancer. The tumor growth was statistically significantly suppressed by CG100649 throughout the 13- to 26-day treatment. Conversely, tumors from CG100649-treated mice showed higher TUNEL staining than tumors from untreated mice (for control tumors, $14.88 \pm 4.7 / \mathrm{high}$-power field (HPF) and for CG100649 treated tumors, 28.7 \pm 7.6/HPF; TUNEL-positive cells, respectively; p<0.001) (Fig. 3D).

CG100649 upregulates the expression of TRAIL receptors (DR4 and DR5) but decreases the expression of decoy receptors (DcR1 and DcR2) in colon cancer. It was previously reported that COX-2 inhibitors increased TRAIL-induced apoptosis and upregulated TRAIL receptors in carcinoma cells $(15,16)$. Therefore, in the present study, we determined whether CG100649 also regulates TRAIL receptors in colon cancer cells. qPCR was performed to examine the 


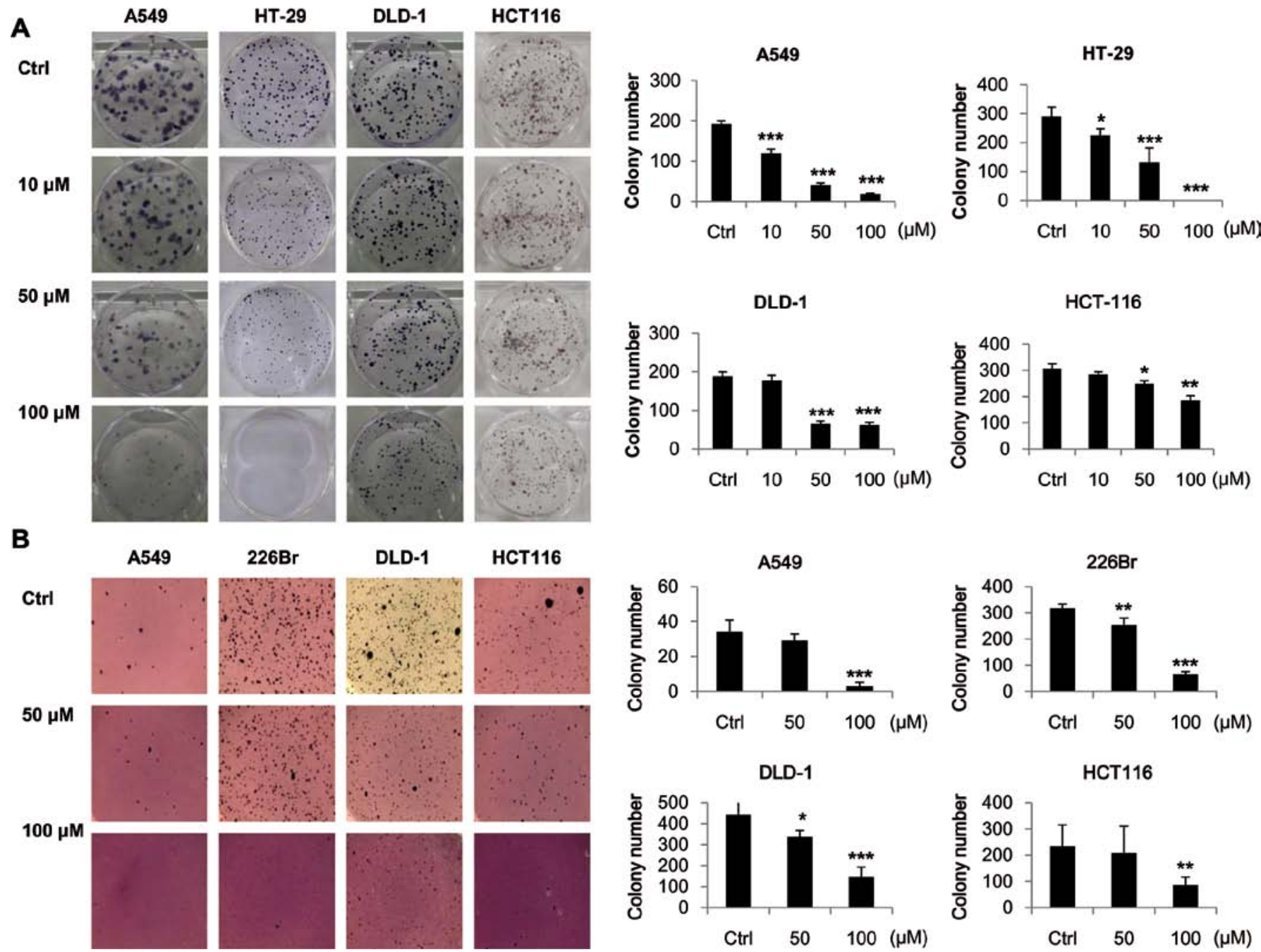

Figure 1. CG100649 inhibits clonogenic survival of human colon and lung cancer cells. (A) Colon and lung cancer cells (5x10² cells/well) were cultured with indicated concentrations of CG100649 or vehicle and maintained for 10 days. Plates were stained with hematoxylin and the colony number was counted. (B) Colon and lung cancer cells ( $1 \times 10^{3}$ cells/well) were suspended in $0.6 \%$ soft agar that was layered on top of $1 \%$ solidified agar in each well of 12 -well plates. At the end of the experiment, tumor cell colonies were counted under a dissection microscope. The bar graph (left panel) shows the mean number of colonies. Data are shown as the means of three independent experiments [error bars show the standard deviation (SD)] ( $\mathrm{p}<0.05,{ }^{* *} \mathrm{p}<0.01$ and $\left.{ }^{* * *} \mathrm{p}<0.001\right)$.
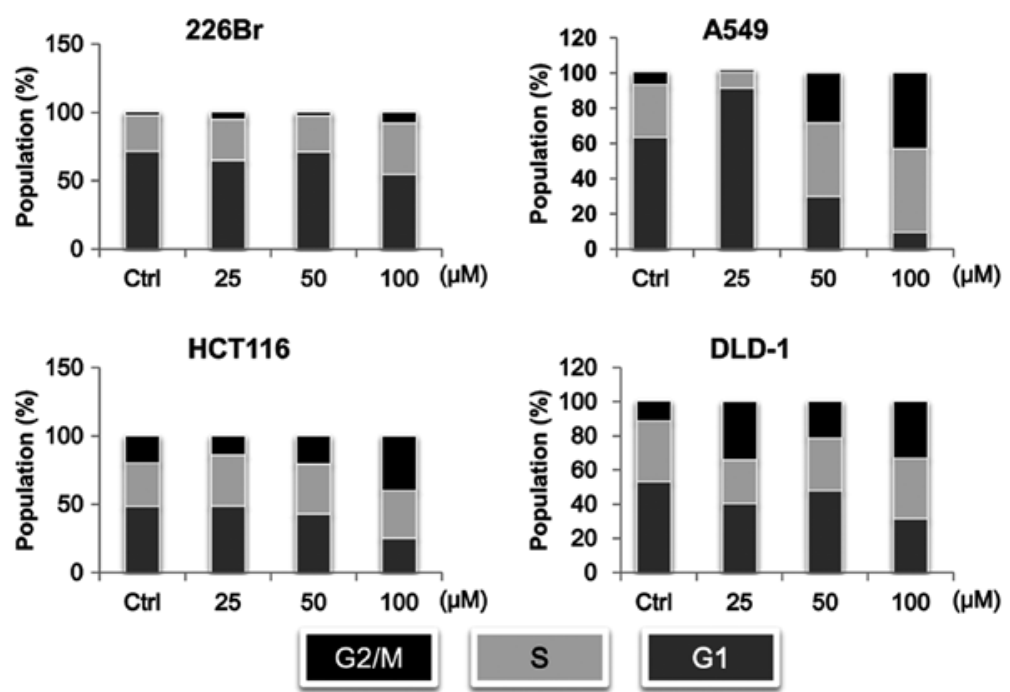

Figure 2. CG100649 induces G2/M cell cycle arrest. The effect of CG100649 on the cell cycle was analyzed by flow cytometry. The treatment of cells with CG100649 induced the G2/M-phase cell cycle arrest in a dose-dependent manner. Colon and lung cancer cells were treated with indicated concentrations of CG100649 or vehicle for $48 \mathrm{~h}$. Propidium iodide-stained cells were analyzed by flow cytometry regarding their DNA content. Representative histograms are shown. Histograms indicate the distribution of DNA content in the CG100649-treated cell population. 
A

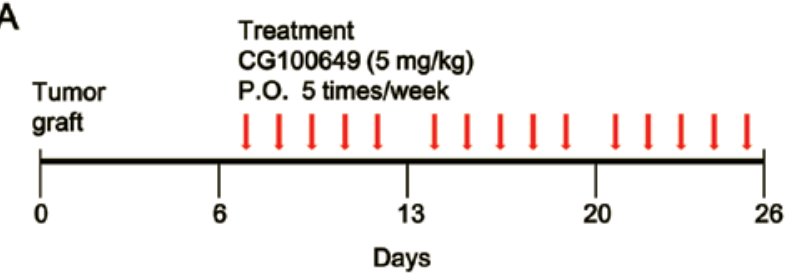

B

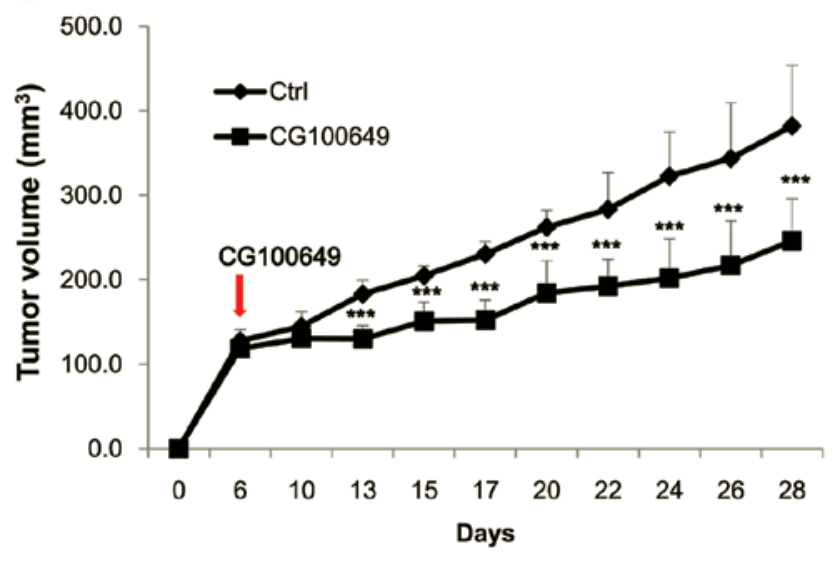

C

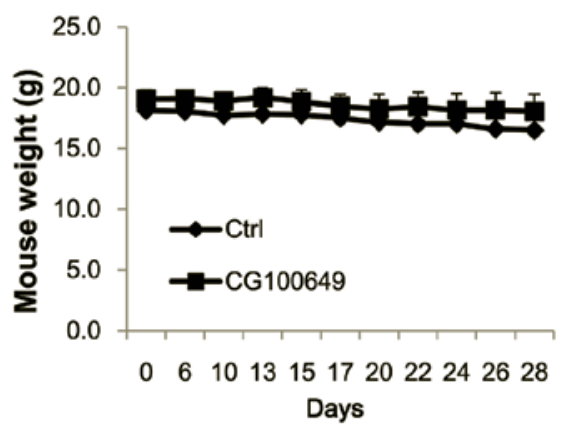

D

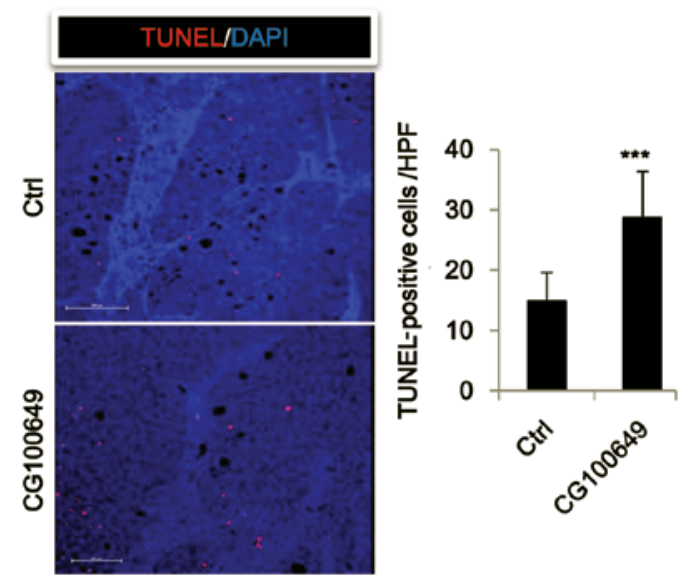

Figure 3. CG100649 inhibits xenograft tumor growth in BALB/c nude mice. (A) Diagram shows the treatment schedule for CG100649. CG100649 (5 mg/kg) or the vehicle was administered orally 5 times a week. After 26 days the mice were sacrificed and the tumors were removed and analyzed. (B) Tumor volumes in animals treated with CG100649 or vehicle were determined as described in 'Materials and methods'. The tumor sizes in animals treated with CG100649 were significantly decreased compared to the untreated group on day $13\left(^{* * *} \mathrm{p}<0.05\right)$. (C) Mouse body weight of tumor-bearing mice treated with vehicle or CG100649. (D) TUNEL assays were performed to observe apoptosis. Apoptotic cells were counted from at least three random fields in tumors. The histogram shows the quantification of TUNEL-positive cells. Significant differences were evaluated using an unpaired two-tailed Student's t-test. [Error bars denote the standard deviation $(\mathrm{SD})]\left({ }^{* * *} \mathrm{p}<0.001\right)$.

expression of TRAIL receptors mRNA in colon cells treated with CG100649. In Fig. 4, a significant increase in DR4 and DR5 mRNA expression was observed in CG100649-treated cells compared with the controls (Fig. 4A). The induction of DR4 and DR5 mRNA by CG100649 was dose-dependent. Furthermore, a statistically significant decrease in DcR1 and DcR2 mRNA expression was observed in CG100649-treated cells (Fig. 4B). We also confirmed the protein expression levels of the TRAIL receptors on the surface of colon cancer cella, following treatment with CG100649, using FACS analysis of extracellular staining (Fig. 4C and D). This indicates that CG100649 may sensitize colon cancer cells to TRAIL-induced apoptosis. DR4 levels increased following treatment with CG100649 in different colon cancer cells, consistent with the qPCR results. DR4 was upregulated by CG100649 treatment, while DR5 was also upregulated in different colon cancer cells by CG100649 treatment (Fig. 4D). The death receptors showed significant upregulation in colon cancer cells, which was induced by CG100649 treatment in a dose-dependent manner.

Treatment with a combination of CG100649 and TRAIL causes enhanced growth suppression in vitro through apoptotic cell death. Having determined that DR4 and DR5 expression was enhanced following exposure to CG100649 in colon cancer cells tested, we determined whether a combination of its ligand, TRAIL, with the CG100649 enhanced drug efficacy. A combination of CG100649 and TRAIL significantly induced substantial cell growth suppression (Fig. 5). Colon cancer cells exhibited a relatively delayed response to CG100649 as determined by the MTT assay (Fig. 5A). Slight growth inhibition was observed at a high dose $(100 \mu \mathrm{M})$ of CG100649 in HCT116 (<30\%) and DLD-1 (<20\%) colon cancer cells. When cells were exposed to TRAIL alone, no effect was observed. By contrast, the combination of CG100649 and TRAIL resulted in a significant decrease in cell growth at lower concentrations $(50 \mu \mathrm{M})$ of CG100649 in HCT116 (>30\%) and DLD-1 (>20\%) as compared to the vehicle control. The administration of $100 \mu \mathrm{M}$ CG100649 with TRAIL as combinatorial treatment achieved 62.7, 61.4 and $42.3 \%$ of cell growth suppression in HCT116, DLD-1 and HT-29 colon cancer cells, respectively. Similarly, $100 \mu \mathrm{M}$ of CG100649 alone produced 29.9, 17.0 and $0.0 \%$ of maximal reduction in cell proliferation. Similar results were obtained using Annexin V/PI staining as a measure of apoptotic cell death. In Fig. 5B, CG100649 combined with TRAIL resulted in a significant increase of apoptotic cell death as compared to the vehicle control, CG100649 or TRAIL as monotherapy. The results revealed that the percentage of cells undergoing apoptosis was increased in the combinatorial treatment: HCT116 ranging from $0.5 \%$ in vehicle control to $0.7,0.6$ and $13.3 \%$ in cells treated with TRAIL, $100 \mu \mathrm{M}$ CG1006491 and $100 \mu \mathrm{M}$ CG1006491 with TRAIL treatment, respectively; DLD-1 
A

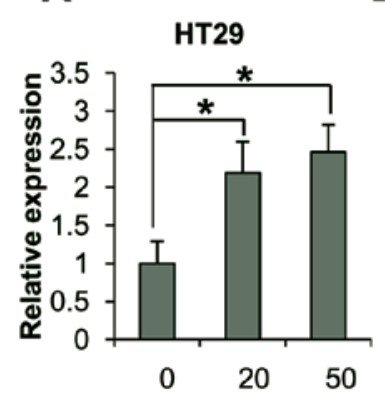

DR4

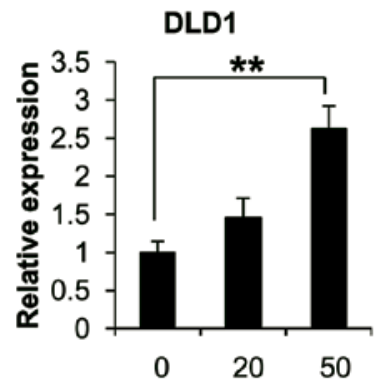

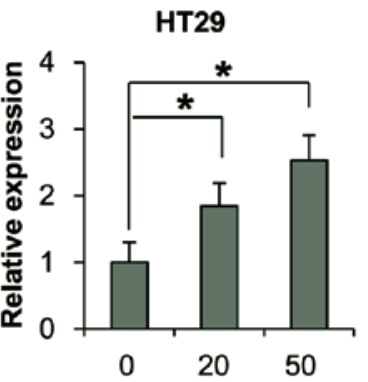

DR5

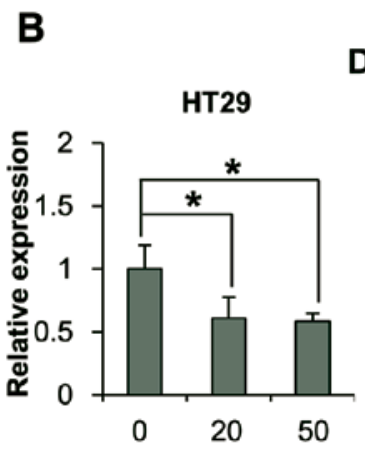

DcR1
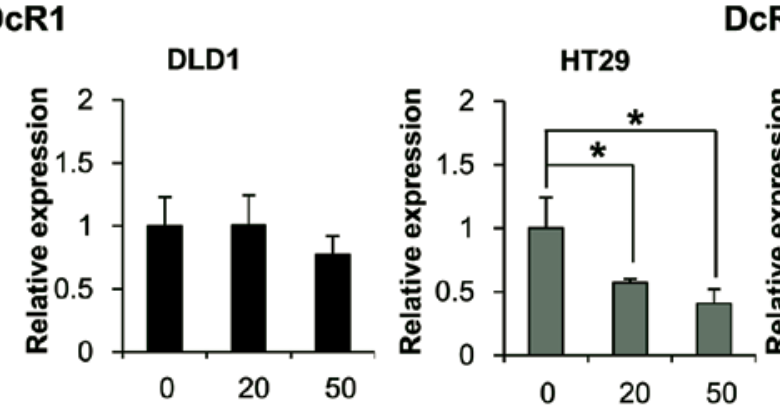

\section{DcR2}

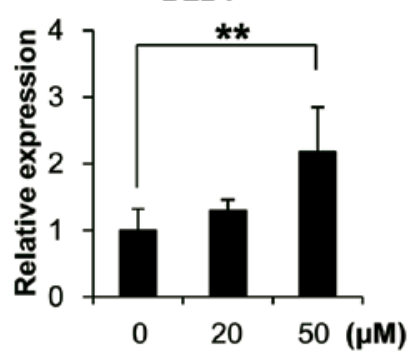

C

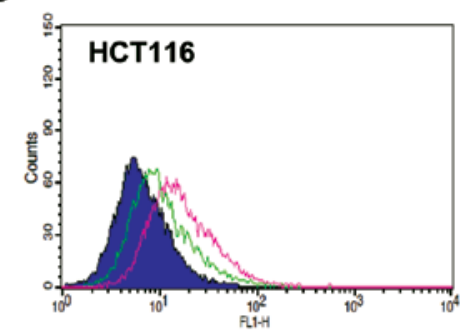

DR4
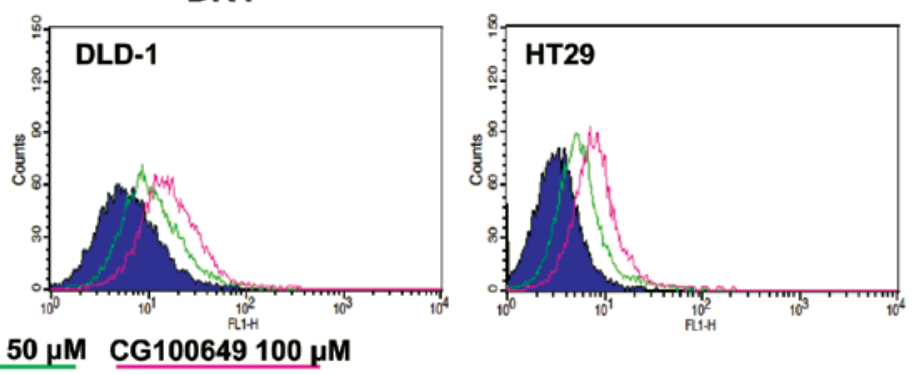

Isotype Control CG100649 $50 \mu \mathrm{M}$ CG100649 $100 \mu \mathrm{M}$

D

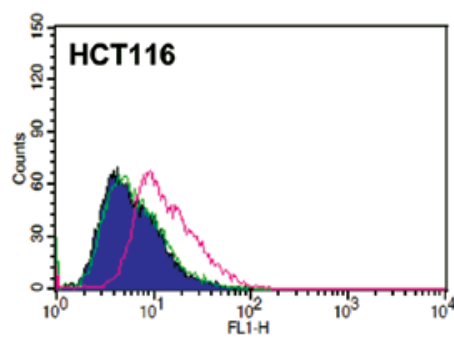

DR5

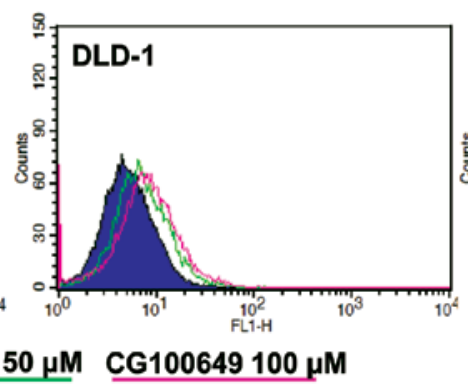

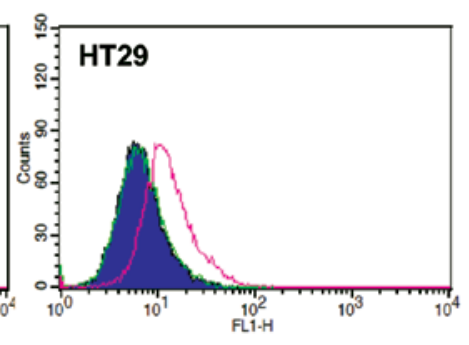

Isotype Control CG100649 $50 \mu \mathrm{M}$ CG100649 $100 \mu \mathrm{M}$

Figure 4. CG100649 upregulated the mRNA expression of TRAIL receptors (DR4 and DR5) but decreased the expression of decoy receptors (DcR1 and DcR2) in colon cancer cells. Colon cancer cells (HT29 and DLD1) were treated with CG100649 (20 and $50 \mu \mathrm{M})$ or vehicle for $48 \mathrm{~h}$. Total RNA was subjected to qPCR. (A) DR4 and DR5 mRNA expression was measured in total RNA isolates by qPCR. (B) The expression of DcR1 and DcR2 was measured by qPCR. Normalized mRNA values were derived by dividing the mRNA value of each target gene with the corresponding quantity of actin mRNA. The cell surface expression of (C) DR4 and (D) DR5 was analyzed by FACS after staining with respective antibodies. FACS histograms are shown: A shift to the right of fluorescence intensity corresponds to an increase in DR4 and DR5 expression at the cell surface. Significant differences were evaluated using an unpaired two-tailed Student's t-test. [Error bars show the standard deviation (SD)] ${ }^{*} \mathrm{p}<0.05$ and $\left.{ }^{* *} \mathrm{p}<0.01\right)$.

ranging from $4.6 \%$ in the vehicle control to $3.8,5.5$ and $17.7 \%$ in cells treated with TRAIL, $100 \mu \mathrm{M}$ CG1006491 and $100 \mu \mathrm{M}$ CG1006491 with TRAIL treatment, respectively; and HT-29 ranging from $7.8 \%$ in vehicle control to $7.1,11.9$ and $38.1 \%$ in cells treated with TRAIL, $100 \mu \mathrm{M}$ CG1006491 and $100 \mu \mathrm{M}$ CG1006491 with TRAIL treatment, respectively. Collectively, these data provided strong evidence that the CG100649 in the present study exerted a pro-apoptotic function independently of its ability to suppress COX-2 activity or carbonic anhydrase (CA)-I/-II activity, and was identified as a particular target in the death receptor pathway.

Combination treatment with CG100649 and TRAIL represses intestinal polyp formation in $A P C^{\text {min/+ }}$ mice. We investigated whether CG100649 or TRAIL prevents intestinal tumorigenesis in $\mathrm{APC}^{\text {min/+ }}$ mice. The experimental schedule is shown 
A

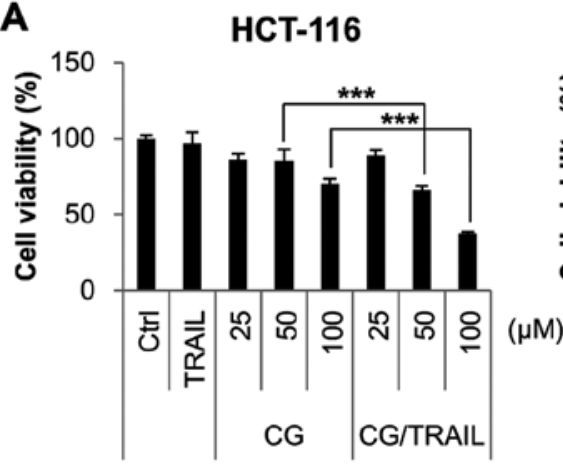

B
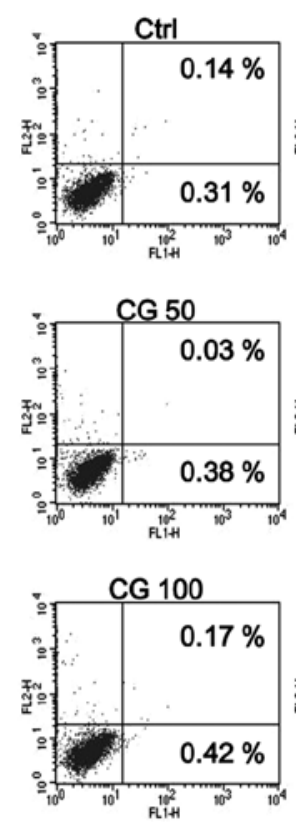

HCT-116
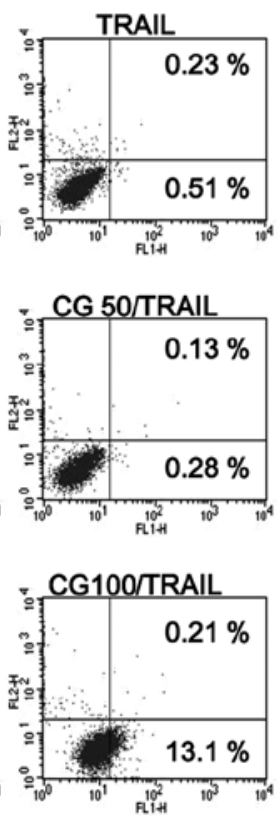

DLD-1
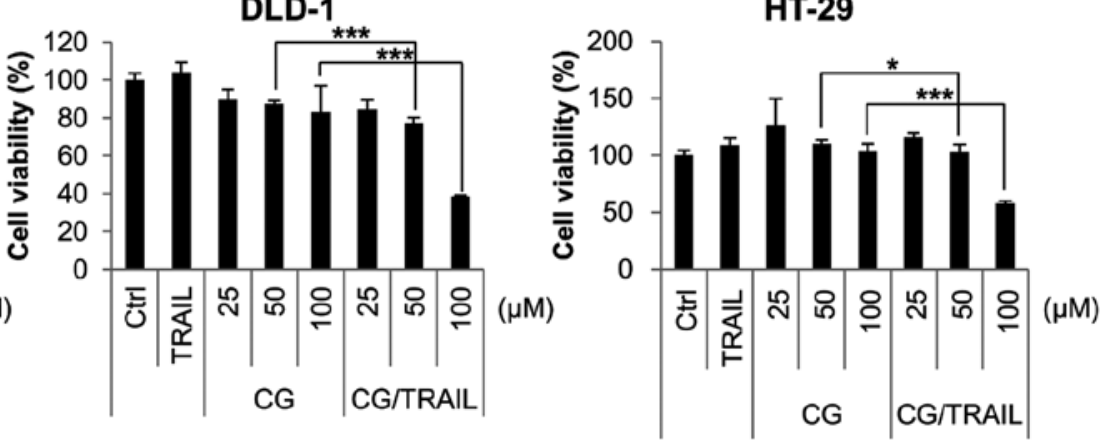

DLD-1
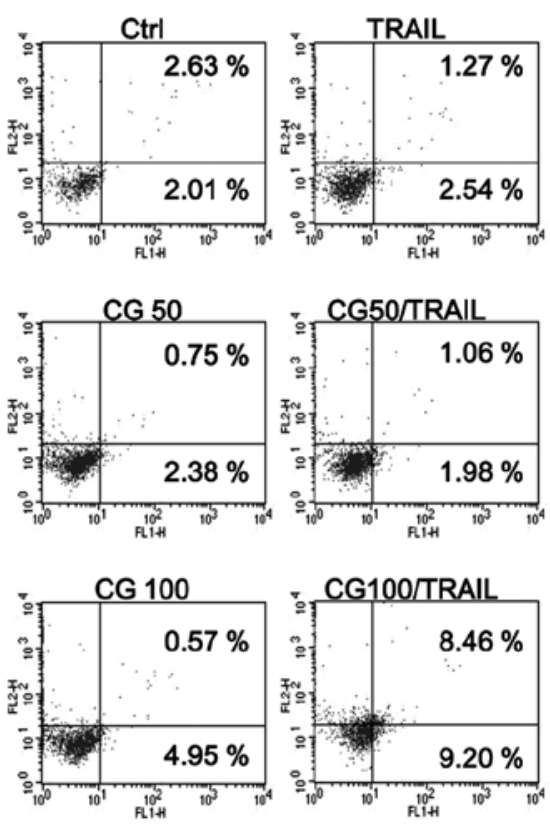

HT-29
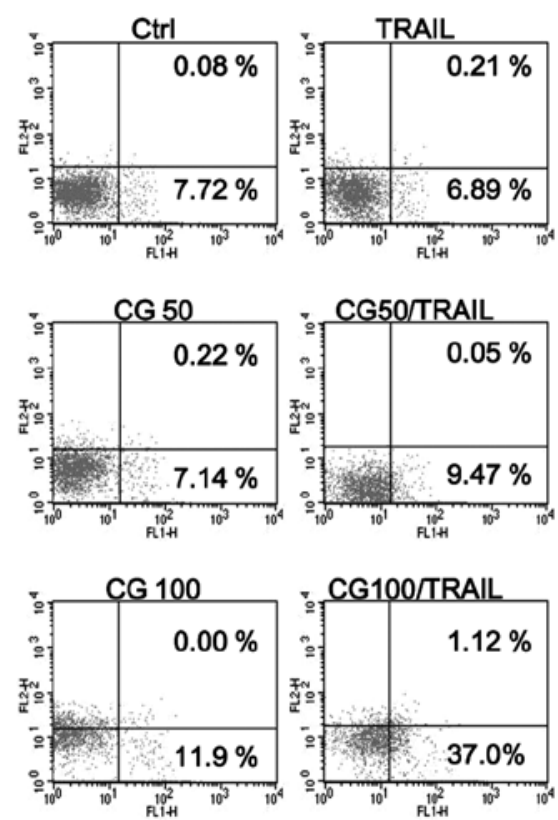

Figure 5. Combination treatment with CG100649 plus TRAIL-induced cell growth suppression and apoptotic cell death in colon cancer cells. (A) Proliferation inhibition assay on colon cancer cells. Cells were treated with vehicle control, CG100649, TRAIL or CG100649 plus TRAIL for 48 h and viability was evaluated by MTT assay. (B) Flow cytometric analysis of Annexin V/PI staining. The cells were plated and exposed to the vehicle control, CG100649, TRAIL or CG100649 plus TRAIL for $48 \mathrm{~h}$. Annexin V and Annexin V/PI double-stained cells were measured by flow cytometry. Significant differences were evaluated using an unpaired two-tailed Student's t-test. [Error bars show the standard deviation (SD)] ( $\left.{ }^{*} \mathrm{p}<0.05,{ }^{* * * *} \mathrm{p}<0.001\right)$.

in Fig. 6A. The co-treatment with CG100649 and TRAIL of $\mathrm{APC}^{\mathrm{min} /+}$ mice resulted in a strong inhibition in intestinal tumorigenesis in terms of decreased polyp number, size and appearance in the small intestine (Fig. 6B). By contrast, CG100649 or TRAIL as monotherapy was unable to reduce intestinal polyp numbers in $\mathrm{APC}^{\mathrm{min} /+}$ mice. On the other hand, the treatment of mice with a combination of CG100649 and TRAIL showed significantly decreased polyp numbers compared to that of mice treated with CG100649 alone. These data are in agreement with our xenograft data (Fig. 3). Our data demonstrated that the combination of TRAIL and CG100649 specifically induced apoptosis and effectively suppressed tumor growth in polyps of $\mathrm{APC}^{\mathrm{min} /+}$ mice. We examined in vivo the effects of CG100649 on DR4 and DR5 by immunohistochemistry in tissues derived from $\mathrm{APC}^{\mathrm{min} /+}$ mice (Fig. 6C). The treatment of mice with CG100649 enhanced the expression of DR4 and DR5. TRAIL as monotherapy exerted no significant effect on the expression of DR4 and DR5. On the other hand, the treatment of mice with a combination of CG100649 and
TRAIL showed a significantly higher expression of DR4 and DR5 compared to that of mice treated with CG100649 or TRAIL as monotherapy. An examination of tumor tissues by TUNEL assay revealed apoptosis induced by CG100649 and TRAIL (Fig. 6D). The TUNEL assay staining analysis revealed a statistically significant increase in apoptosis induced by a combination of CG100649 and TRAIL (control, 14.3 \pm 12.7 ; TRAIL, 43 \pm 17.6 ; CG100649, 203.7 \pm 48.4 ; and combination, 364.5 \pm 95.8 TUNEL-positive cells, respectively; $\mathrm{P}<0.001)$. These data suggested that CG100649 sensitized colon cancer cells to TRAIL treatment in $\mathrm{APC}^{\text {min/+ }}$ mice.

\section{Discussion}

In the present study, we investigated the anticancer activity of CG100649 and its action mechanism in human colon cancer cells. CG100649 inhibited anchorage-dependently and -independently the colony formation in human colon and lung cancer cells suggesting that the compound suppresses 


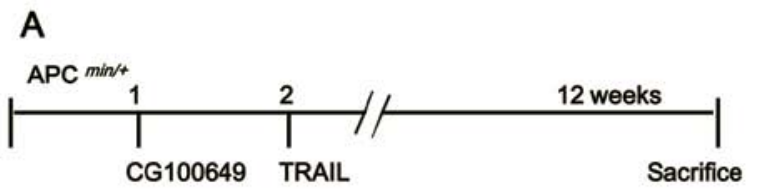

TRAIL (3 mg/kg; by I.P.; Twice/week)

CG100649 (1 mg/kg; by P.O.; Once/day)

B

Female APC $\min /+$
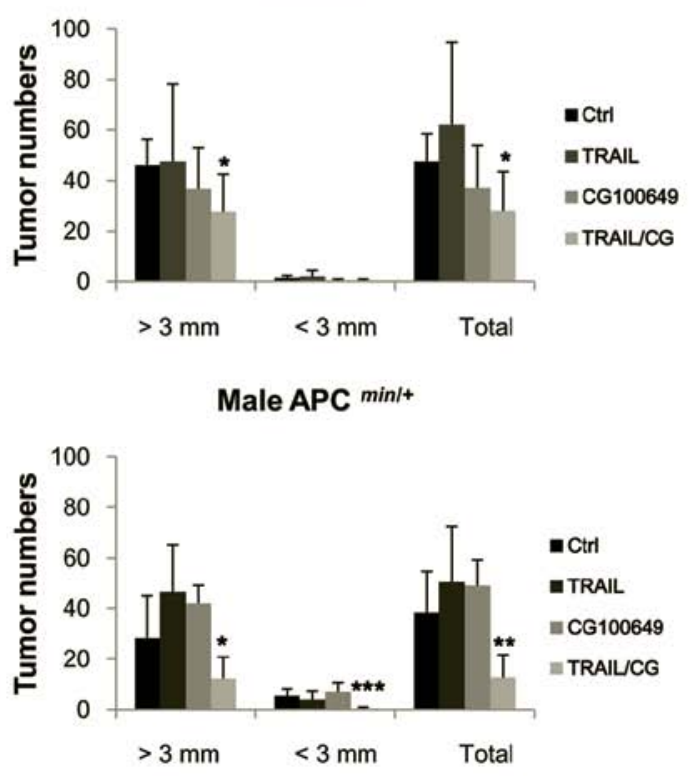

D
C

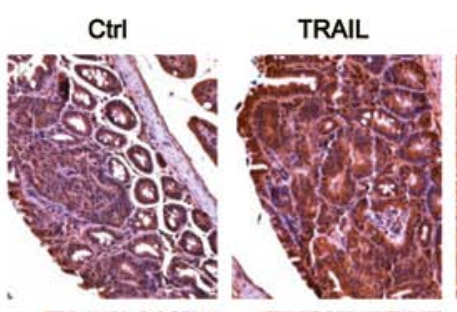

DR5
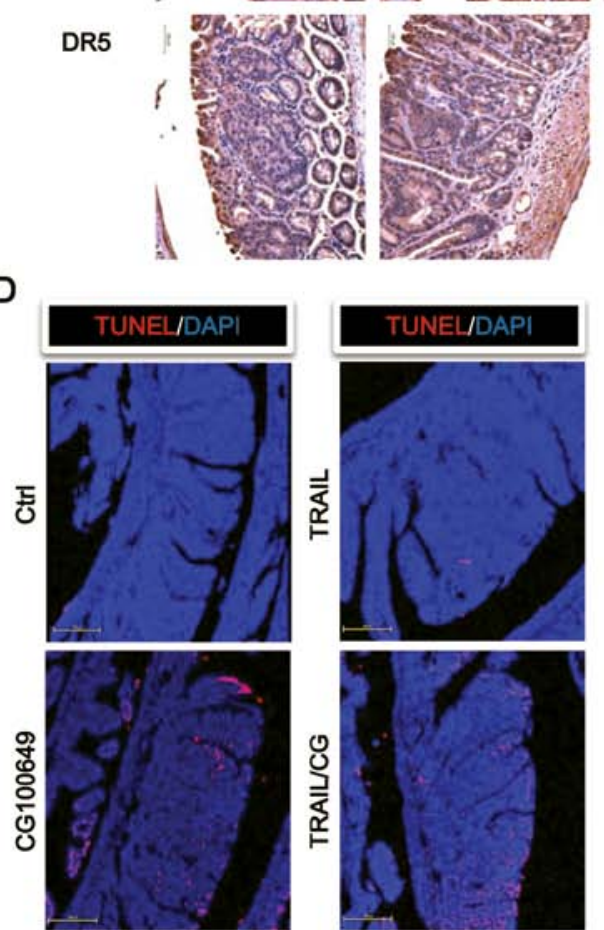

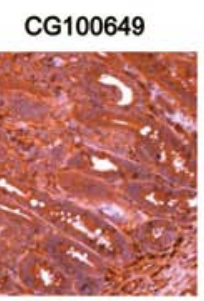

TRAIL/CG
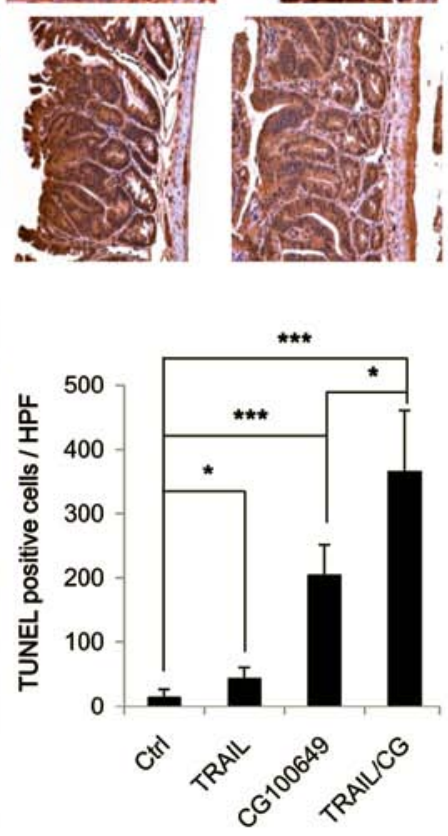

Figure 6. Co-treatment with CG100649 and TRAIL represses intestinal polyp formation in APC ${ }^{\text {min/+ }}$ mice. (A) Experimental schedule for TRAIL and CG100649 combination treatment in $\mathrm{APC}^{\mathrm{min} /+}$ mice. (B) At the end of the experiment, results in the small intestine are shown for the number of polyps/mice. (C) Immunohistochemical features of the small intestine tissues. Formalin-fixed paraffin sections were stained with anti-DR4 and anti-DR5 antibodies. (D) Tunnel assays were performed to observe apoptosis. Apoptotic cells were counted from at least three random fields in tumors. The histogram shows the quantification of TUNEL-positive cells. Significant differences were evaluated using an unpaired two-tailed Student's t-test. [Error bars show the standard deviation (SD)] $\left({ }^{*} \mathrm{p}<0.05\right.$ and $\left.{ }^{* *} \mathrm{p}<0.01\right)$.

colon and lung cancer survival. The inhibition induced by CG100649 was associated with an increase in the G2/M phase cell population and a decrease in the G1 phase cell population in colon and lung cancer cells. The G2/M DNA damage checkpoint serves to prevent the cell from entering mitosis (M-phase) with genomic DNA damage (17). Specifically, the activity of the cyclin B-cdc2 (CDK1) complex is important in regulating the G2-phase transition wherein cdc2 is maintained in an inactive state by the tyrosine kinases Weel and Myt1 (18). Microtubules play an important role in the regulation of the mitotic processes and their disruption can induce cell-cycle arrest in the $\mathrm{M}$ phase as well as the formation of abnormal mitotic spindles and apoptotic cell death (19).

Notably, CG100649 inhibited tumor growth in colon cancer xenografts in nude mice, the tumor volume was decreased in mice treated with CG100649 as compared to control. This suggests therapeutic activity of CG100649 for colon cancer. It was reported that lycopene and fish oil act synergistically as chemopreventive agents against tumor growth and progression in a mouse xenograft model of colon cancer (20). A doubleblind, randomized, multicenter, active- and placebo-controlled phase III study (?) was performed to evaluate the efficacy and safety of CG100649 in osteoarthritis patients. The present study demonstrates that CG100649 may be used to assess its efficacy and safety in colon cancer patients.

Previous findings have shown that a variety of chemotherapeutic drugs, including cisplatin, etoposide and doxorubicin, can sensitizes a wide range of cancer cells to TRAIL-induced apoptosis $(15,21,22)$. We studied the relationship between CG100649 and TRAIL to investigate the mechanism by which CG100649 suppresses tumor growth. It was reported that COX-2 inhibitors increased TRAIL-induced apoptosis and upregulated TRAIL receptors in hepatocellular carcinoma cells (15). It was also reported that the inhibition of COX-2 expression by topical given NSAID such as diclofenac enhanced the radiation sensitivity via enhancement of TRAIL in a human prostate adenocarcinoma xenograft model (23). Martin et al reported that COX-2 inhibition sensitizes human colon carcinoma cells to TRAIL-induced apoptosis by clustering of DR5 and concentrating death-inducing signaling complex components into ceramide-enriched caveolae (16). Therefore, we evaluated whether CG100649 regulates TRAIL receptors and sensitizes colon cancer to TRAIL treatment. We found that CG100649 upregulated DR4 and DR5 mRNA levels but downregulated 
DcR1 and DcR2 mRNA levels. Activation of DR4 and DR5 was also regulated by the level of expression of decoy receptors such as DcR1 and DcR2 that bind to TRAILs instead of DR4 and DR5 and inhibit apoptotic pathways $(24,25)$. In addition, the cell surface upregulation of DR4 and DR5 was confirmed by FACS analysis. These data show that CG100649 induces TRAIL receptor expression in cancer cells. We also examined whether a combination of its ligand, TRAIL with CG100649 enhanced cytotoxicity. The viability and apoptotic cell death were measured following monotherapy or combinatorial treatment. Treatment with CG100649 plus TRAIL effectively and synergistically suppressed cell growth and enhanced apoptotic cell death. Upregulation of TRAIL-induced apoptosis by CG100649 was due to the increased membrane expression of TRAIL receptors. Enhanced expression of TRAIL receptors at the cell surface by CG100649 has been shown to increase sensitivity to TRAIL.

The results show that CG100649 assists the activity of TRAIL inhibiting intestinal tumorigenesis in terms of decreased polyp number, size and appearance in the small intestine in $\mathrm{APC}^{\mathrm{min/+}}$ mice. This finding suggests that CG100649 contains chemopreventive activity for colon cancer. There is interest in cancer therapy producing cell death by activation of the TRAIL receptor-mediated apoptotic pathway while avoiding decoy-receptor-mediated neutralization of the signal. This facilitated the development of various receptor-specific TRAIL variants and agonistic antibodies. Such recombinant TRAIL and agonist antibodies targeting TRAIL-R1/DR4 and/or TRAIL-R2/DR5 are currently under investigation in clinical trials. In addition, TRAIL-resistant tumors may be sensitized to TRAIL by a combination of TRAIL or agonistic antibodies with chemotherapeutic agents, targeted small molecules or irradiation (26).

In conclusion, results of the present study demonstrate that CG100649 contains preventive and therapeutic activity for colon cancer. This finding may be useful in the identification of the mechanism of colon cancer development and in the role of CG100649.

\section{Acknowledgements}

This study was supported by a grant from the Global Core Research Center (GCRC) grant (no. 2011-0030678) from NRF, MEST, Republic of Korea.

\section{References}

1. Siegel R, Naishadham D and Jemal A: Cancer statistics, 2013 CA Cancer J Clin 63: 11-30, 2013.

2. Kim MK, Kang YJ, Kim DH, et al: A novel hydroxamic acid derivative, MHY218, induces apoptosis and cell cycle arrest through downregulation of NF- $\kappa \mathrm{B}$ in HCT116 human colon cancer cells. Int J Oncol 44: 256-264, 2014.

3. Gurpinar E, Grizzle WE and Piazza GA: NSAIDs inhibit tumorigenesis, but how? Clin Cancer Res 20: 1104-1113, 2014.

4. Coghill AE, Newcomb PA, Chia VM, et al: Pre-diagnostic NSAID use but not hormone therapy is associated with improved colorectal cancer survival in women. Br J Cancer 104: 763-768, 2011.

5. Skarke C, Alamuddin N, Lawson JA, Cen L, Propert KJ and Fitzgerald GA: Comparative impact on prostanoid biosynthesis of celecoxib and the novel nonsteroidal anti-inflammatory drug CG100649. Clin Pharmacol Ther 91: 986-993, 2012.
6. Shankar S, Singh TR, Fandy TE, Luetrakul T, Ross DD and Srivastava RK: Interactive effects of histone deacetylase inhibitors and TRAIL on apoptosis in human leukemia cells: Involvement of both death receptor and mitochondrial pathways. Int J Mol Med 16: 1125-1138, 2005.

7. Shankar S, Ganapathy S, Chen Q and Srivastava RK: Curcumin sensitizes TRAIL-resistant xenografts: molecular mechanisms of apoptosis, metastasis and angiogenesis. Mol Cancer 7: 16, 2008.

8. Shankar S and Srivastava RK: Enhancement of therapeutic potential of TRAIL by cancer chemotherapy and irradiation: mechanisms and clinical implications. Drug Resist Updat 7: 139-156, 2004.

9. Ren YG, Wagner KW, Knee DA, Aza-Blanc P, Nasoff M and Deveraux QL: Differential regulation of the TRAIL death receptors DR4 and DR5 by the signal recognition particle. Mol Biol Cell 15: 5064-5074, 2004.

10. Liu XS, Zhu Y, Han WN, et al: Preparation and characterization of a set of monoclonal antibodies to TRAIL and TRAIL receptors DR4, DR5, DcR1, and DcR2. Hybrid Hybridomics 22: 121-125, 2003.

11. Krishan A: Rapid flow cytofluorometric analysis of mammalian cell cycle by propidium iodide staining. J Cell Biol 66: 188-193, 1975.

12. Oh SH, Woo JK, Jin Q, et al: Identification of novel antiangiogenic anticancer activities of deguelin targeting hypoxia-inducible factor-1 alpha. Int J Cancer 122: 5-14, 2008.

13. Oh SH, Woo JK, Yazici YD, et al: Structural basis for depletion of heat shock protein 90 client proteins by deguelin. J Natl Cancer Inst 99: 949-961, 2007.

14. Han JY, Oh SH, Morgillo F, et al: Hypoxia-inducible factor $1 \alpha$ and antiangiogenic activity of farnesyltransferase inhibitor SCH66336 in human aerodigestive tract cancer. J Natl Cancer Inst 97: 1272-1286, 2005.

15. Yamanaka Y, Shiraki K, Inoue T, et al: COX-2 inhibitors sensitize human hepatocellular carcinoma cells to TRAIL-induced apoptosis. Int J Mol Med 18: 41-47, 2006.

16. Martin S, Phillips DC, Szekely-Szucs K, Elghazi L, Desmots F and Houghton JA: Cyclooxygenase-2 inhibition sensitizes human colon carcinoma cells to TRAIL-induced apoptosis through clustering of DR5 and concentrating death-inducing signaling complex components into ceramide-enriched caveolae. Cancer Res 65: 11447-11458, 2005.

17. Cuddihy AR and O'Connell MJ: Cell-cycle responses to DNA damage in G2. Int Rev Cytol 222: 99-140, 2003.

18. Zhang XH, Zou ZQ, Xu CW, Shen YZ and Li D: Myricetin induces $\mathrm{G} 2 / \mathrm{M}$ phase arrest in HepG2 cells by inhibiting the activity of the cyclin B/Cdc2 complex. Mol Med Rep 4: 273-277, 2011.

19. Nicolini F, Burmistrova O, Marrero MT, et al: Induction of $\mathrm{G}_{2} / \mathrm{M}$ phase arrest and apoptosis by the flavonoid tamarixetin on human leukemia cells. Mol Carcinog 53: 939-950, 2014.

20. Tang FY, Pai MH, Kuo YH and Wang XD: Concomitant consumption of lycopene and fish oil inhibits tumor growth and progression in a mouse xenograft model of colon cancer. Mol Nutr Food Res 56: 1520-1531, 2012.

21. Lacour S, Hammann A, Wotawa A, Corcos L, Solary E and Dimanche-Boitrel MT: Anticancer agents sensitize tumor cells to tumor necrosis factor-related apoptosis-inducing ligandmediated caspase- 8 activation and apoptosis. Cancer Res 61: 1645-1651, 2001.

22. Xu LH, Deng CS, Zhu YQ, Liu SQ and Liu DZ: Synergistic antitumor effect of TRAIL and doxorubicin on colon cancer cell line SW480. World J Gastroenterol 9: 1241-1245, 2003.

23. Inoue $\mathrm{T}$, Anai $\mathrm{S}$, Onishi $\mathrm{S}$, et al: Inhibition of COX-2 expression by topical diclofenac enhanced radiation sensitivity via enhancement of TRAIL in human prostate adenocarcinoma xenograft model. BMC Urol 13: 1, 2013.

24. Sheridan JP, Marsters SA, Pitti RM, et al: Control of TRAILinduced apoptosis by a family of signaling and decoy receptors. Science 277: 818-821, 1997.

25. Pan G, Ni J, Wei YF, Yu G, Gentz R and Dixit VM: An antagonist decoy receptor and a death domain-containing receptor for TRAIL. Science 277: 815-818, 1997.

26. Abdulghani J and El-Deiry WS: TRAIL receptor signaling and therapeutics. Expert Opin Ther Targets 14: 1091-1108, 2010. 